\title{
C
}

\section{British Ecological Society}

Spatially Induced Bifurcations in Single-Species Population Dynamics

Author(s): Jordi Bascompte and Ricard V. Solé

Reviewed work(s):

Source: Journal of Animal Ecology, Vol. 63, No. 2 (Apr., 1994), pp. 256-264

Published by: British Ecological Society

Stable URL: http://www.jstor.org/stable/5544

Accessed: 16/01/2012 07:42

Your use of the JSTOR archive indicates your acceptance of the Terms \& Conditions of Use, available at http://www.jstor.org/page/info/about/policies/terms.jsp

JSTOR is a not-for-profit service that helps scholars, researchers, and students discover, use, and build upon a wide range of content in a trusted digital archive. We use information technology and tools to increase productivity and facilitate new forms of scholarship. For more information about JSTOR, please contact support@ jstor.org. 


\author{
JORDI BASCOMPTE* and RICARD V. SOLÉ $\dagger$ \\ Complex Systems Research Group, *Departament d'Ecologia, Universitat de Barcelona, Diagonal 645, \\ 08028 Barcelona, Spain; and †Departament de Física i Enginyeria Nuclear, Universitat Politècnica de \\ Catalunya, Pau Gargallo 5, 08028 Barcelona, Spain
}

\begin{abstract}
Summary
1. The paper by Hassell, Lawton \& May (1976) on the characterization of natural population dynamics by fitting field and laboratory data to a discrete model is reviewed. In particular, we study the effects of spatial degrees of freedom on the qualitative behaviour of such single population maps.

2. Using the spatially extended counterpart of these discrete systems by means of the Coupled Map Lattice formalism, we show that space is able to induce dynamical bifurcations.

3. As the spatial domain is increased, the stability boundaries in the parameter space are modified, driving the population through different kinds of dynamics. The same result is reached by increasing the diffusion rate for a given lattice size.

4. Our results suggest that the dynamical behaviour shown by a population depends not only on intrinsic factors, but also on external or environmental ones. Some evidence from field studies is also discussed.
\end{abstract}

Key-words : population dynamics, spatiotemporal chaos, bifurcation, space, ecological models.

Journal of Animal Ecology (1994) 63, 256-264

\section{Introduction}

Since the evidence of May (1974, 1976), and May \& Oster (1976) that simple non-linear discrete models of population biology could show a wide spectrum of complex behaviour, ecologists have had another alternative when trying to understand the nature of complexity in natural systems. Such difference equations are able to generate a complex, aperiodic and randomlike motion (known as deterministic chaos) in spite of being totally deterministic. The next question was: are the erratic fluctuations observed in nature due to noise or is there a deterministic density-dependent mechanism underlying the dynamics? The attempt to answer this question was the classic paper by Hassell, Lawton \& May (1976). The authors collected lifetable data from 28 insect populations with nonoverlapped generations, fitting such data to a simple single-species discrete model. They estimated the parameter values associated with each field and laboratory population and concluded that most of the studied data showed steady state, with only one example of limit cycle or periodic motion and one corresponding to chaos, i.e. the Nicholson blowflies.

The great influence of this paper meant that, as pointed out by Pimm (1991), most field ecologists thought of deterministic chaos as a mathematical possibility not realized by the real world. Other studies using the same kind of approach have reinforced the point of view according to which the 'normal' dynamical behaviour found in laboratory drosophilid species is the stable one and concluding that limit cycles and chaos may be maladaptative. These studies have proposed group selection for stability (Thomas, Pomeranz \& Gilpin 1980) or even selection at the individual level (Mueller \& Ayala 1981). Additional ecological reasoning has reinforced this opposition to chaos (Berryman \& Millstein 1989a). The idea is that chaotic unstable dynamics would facilitate a high probability of extinction (see, however, Nisbet et al., 1984; Lomnicki 1989; Mani 1989 and the reply of Berryman \& Millstein, 1989b for a discussion of the particular mechanism involved in avoiding chaos).

On the other hand, Schaffer \& Knot have subscribed to the point of view according to which chaos may be an important component of ecological systems from their application of dynamical systems tools to field data (Schaffer \& Knot 1986). Some of these conclusions have been reinforced by the use by Sugihara \& May (1991) of non-linear forecasting methods. 
Thus, it seems reasonable to believe that many of the field ecologists have pushed away chaos too early. Due to the great influence noted above of the paper by Hassell, Lawton \& May (1976) in this rejection, it is necessary to reconsider some aspects of that work.

As already pointed out by Hassell, Lawton \& May (1976), their conclusions should be considered with caution because they are based on a single-species model excluding interaction terms with other populations or dispersal patterns. As they recognize, there is at least a case in which the model does not correctly describe the dynamics shown by the population. The moth Zeiraphera diniana appears in their analysis as a monotonically stable population, although it is well known that it shows regular oscillations with a welldefined period of eight generations. There are, in fact, a large number of problems in characterizing the kind of dynamics of a population by fitting simple discrete models to the census data. As pointed out by Morris (1990), the parameter estimation depends largely on the estimation procedure and the particular model used, leading to different conclusions about the same population. Morris (1990) concludes that 'given these limitations, inferences about the presence or absence of chaos in natural insect populations that rely on simple best-fit models are premature'.

The bulk of this paper deals with the analysis of the changes in the dynamical properties of discrete models when spatial degrees of freedom are introduced and how the size of the spatial domain and the dispersion rates modify the stability boundaries, and so inferences about the kind of dynamics shown by a given population when fitting the data to a simple model.

\section{A look at the discrete model}

To begin with, consider the Hassell (1975) singlepopulation, discrete model:

$N_{t+1}=\lambda N_{t}\left(1+\alpha N_{t}\right)^{-\beta}$

$N_{t}, N_{t+1}$ being the respective population densities at successive generations $t$ and $t+1$. $\lambda$ is the net growth rate, and $\alpha$ and $\beta$ are constants defining the densitydependent feedback term.

Model 1 shows a characteristic parameter space with well-defined stability boundaries delimiting the regions of monotonic damping, oscillatory damping, limit cycles and chaos depending on the parameter values. The steady state, i.e. the population level $N^{*}$ such that $F\left(N^{*}\right)=N^{*}$ is given by the expression $N^{*}=\left(\lambda^{1 / \beta}-1\right) / \alpha$. This solution is stable if

$$
\left|\frac{\partial F\left(N^{*}\right)}{\partial N}\right|<1,
$$

and it becomes unstable just when the equality

$$
\frac{\partial F\left(N^{*}\right)}{\partial N}=-1
$$

holds (May \& Oster 1976). For the present case, this leads us to the conclusion that the condition

$\beta\left(1-\lambda^{-1 / \beta}\right)=2$

eqn 2

defines the isocline dividing the stable set from the unstable one. The latter expression can be rewritten in an explicit way as

$\lambda=\exp [-\beta \ln (1-2 / \beta)]$

eqn 3

which allows us to represent the stability boundary in the parameter space. As can be observed, there are only two parameters $(\lambda$ and $\beta$ ) affecting stability.

The Hassell model is used by Hassell, Lawton \& May (1976) to fit the census data. First, they rewrote model 1 in the following way:

$\log \left(\lambda N_{t} / N_{t+1}\right)=\log \left(N_{t} / N_{\mathrm{s}}\right)=\beta \log \left(1+\alpha N_{t}\right) \quad$ eqn 4 where $N_{\mathrm{s}}=N_{t+1} / \lambda$ is the number of surviving larvae in generation $t$, i.e. the number of individuals surviving the density-dependent phase of the life cycle when the number entering this stage is $N_{t} . \beta$ is the slope of the relationship between mortality $\left(\log N_{t} / N_{\mathrm{s}}\right)$ and population size $\left(\log N_{t}\right)$ at high population levels and $\alpha$ is related to the point of inflection of the curve. In order to estimate $\alpha$ and $\beta$ they plot mortality in the density-dependent stage against initial density at that stage using a least-squares technique to fit equation (4) to the data. On the other hand, $\lambda$ is estimated taking into account the maximum per capita replacement rate and subtracking from it the average value of all density-independent mortalities acting during the life cycle (Hassell, Lawton \& May 1976).

In doing so, they represent, in such a parametric space ( $\lambda$ vs. $\beta$ ), the estimated constant values for the different field and laboratory populations (see Fig. 1). As pointed out above, almost all the examples lie in the stable domain.

\section{The spatially extended counterpart}

The introduction of space in discrete temporal maps can be made by means of the Coupled Map Lattice (CML) formalism. A CML is a dynamical system with discrete time, discrete space and continuous state (Kaneko 1989, 1990, 1992; Solé, Bascompte \& Valls 1992a). The nodes of such a discrete space are the elements of the set:

$\mathbf{\Lambda}^{(2)}=\{\mathbf{K}=(i, j) \mid 1 \leqslant i, j \leqslant L\}$

eqn 5

and the corresponding spatiotemporal dynamics for the Hassell map is defined by the following expression :

$N_{t+1}(i, j)=\lambda N_{t}(i, j)\left(1+\alpha N_{t}(i, j)\right)^{-\beta}+D \nabla^{2} N_{t}(r)$ eqn 6

We then define the dispersion between the adjacent patches in a very clear way as a physical diffusion extended to the four nearest neighbours. The discrete laplacian operator is 
258

Spatially induced bifurcations

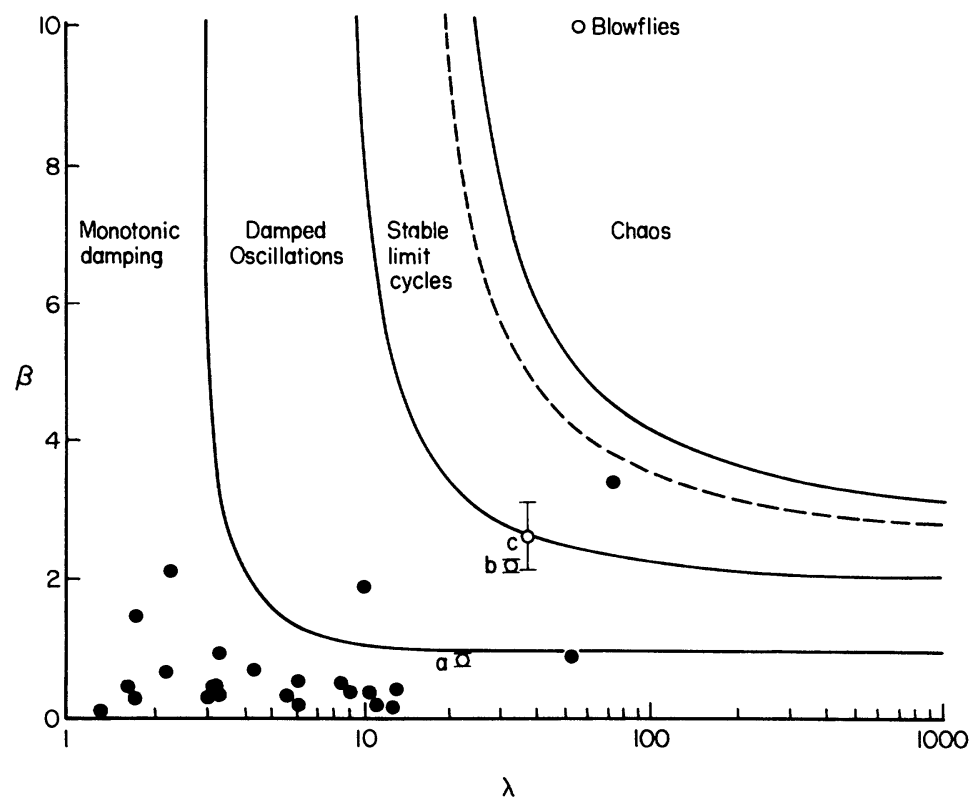

Fig. 1. The parameter space for the Hassell map (equation 1) with the isoclines defining the different stability domains. The points correspond to the least squares fitting of 28 laboratory and field insect time series. After Hassell, Lawton \& May (1976).

$$
\begin{aligned}
& \nabla^{2} N_{t}(r)=N_{t}(i-1, j)+N_{t}(i+1, j) \\
& \quad+N_{t}(i, j-1)+N_{t}(i, j+1)-4 N_{t}(i, j)
\end{aligned}
$$

$D$ being the diffusion rate. An additional rule is used in order to avoid negative values when the system is very unstable, i.e. $N_{t}(i, j)=0$ if $N_{t}(i, j)>0$.

Under these conditions we are able to study the spatial Hassell model (6) making numerical experiments for different parameter combinations and testing the effect of space on the qualitative behaviour of the map. In what follows we are considering the local dynamics, i.e. the dynamics at a given lattice point.

There is a great interest in the relationship between local and global dynamics. In fact, the spatial effects on population dynamics may radically change the conditions of persistence and coexistence as has been noted by many others, e.g. den Boer (1987); Taylor (1990); Sabelis, Diekmann \& Jansen (1991); Hassel, Comins \& May (1991); Czárán \& Bartha (1992); Solé \& Valls (1992); Moloney et al. (1992).

The effect of dispersal on population stability seems to depend on the specific kind of coupling between the patches. Discrete space population models have shown that dispersal can sometimes stabilize and sometimes destabilize populations (Vance 1984). When the dispersal from one patch is a global mixing to all others patches, migration per se neither increases nor decreases stability. However, when the dispersion is reduced to nearest patches, there is a clear stability effect (Hassell, Comins \& May 1991; Solé 1991; Solé \& Valls 1992).

The explanation for this difference in the qualitative behaviour of the two kinds of dispersal comes as follows. As pointed out by Comins, Hassell \& May
(1992), there is a close relationship between the metapopulation stability and the generation and maintenance of patterns in space. Such self-organization is destroyed if there is a global mixing of populations. On the other hand, it is widely present for a large number of parameter combinations if dispersal is limited to the nearest points (Hassell, Comins \& May 1991 ; Solé, Valls \& Bascompte 1992). In our opinion there is here a key idea regarding the relationships between spatial patterns and temporal properties in biological systems. As a consequence, this richness of spatial patterns could have an adaptive meaning. A recent example of the evolutionary implications of such spatial structures is provided by Boerlijst \& Hogeweg (1991).

\section{Results}

We have studied the Hassell CML (6) for different parameter combinations. It has been demonstrated that even for values that correspond to a steady state in the corresponding map (1), enlarging the lattice size yields successive bifurcations leading to periodic and chaotic motion, as seen in the respective temporal series shown in Fig. 2. In other words, space acts as a bifurcation parameter. Such spatial effect is clearly appreciated from Fig. 3, where the bifurcation scenario for the Hassell map has been represented when $\beta$ is made successively higher (Fig. 3a) and for CML counterparts of lattice size, respectively $4 \times 4$ (Fig. 3b) and $5 \times 5$ (Fig. 3c). As noted, all the bifurcations appear earlier, i.e. for lower $\beta$-values for the CML counterparts in relation with the uncoupled map. Furthermore, the longer the spatial domain, the wider 
259

J. Bascompte \& R.V. Solé
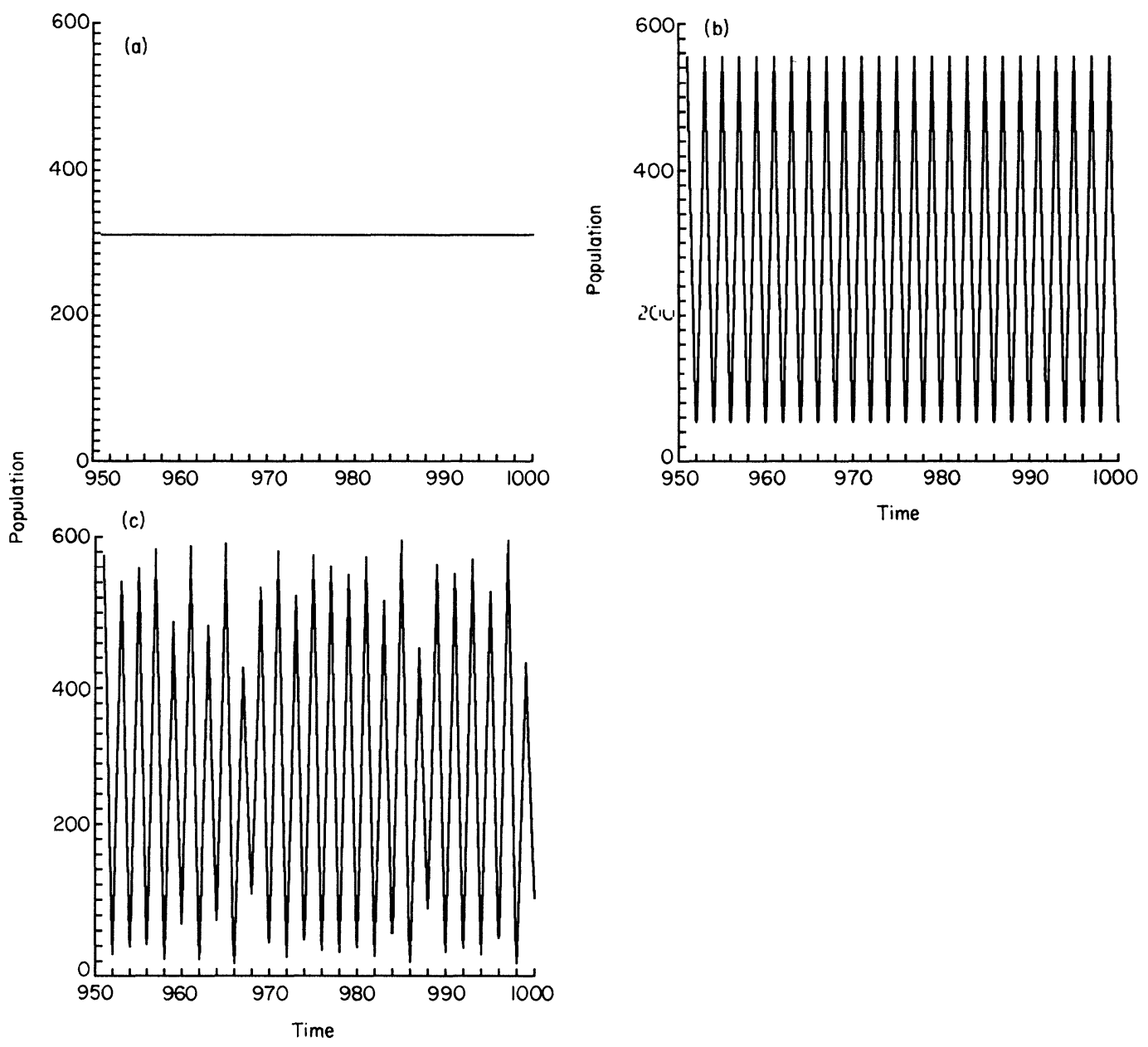

Fig. 2. Spatially induced bifurcations. The dynamics for the Hassell map (equation 1) is shown with $\lambda=30, \alpha=0.01$ and $\beta=2.7$ corresponding to a steady state (a), and the same parameter combinations, but for the CML (equation 6) with $D=0.05$ and lattice size $4 \times 4$ (b) and $11 \times 11$ (c), respectively. As noted, the size of the spatial domain is important for the characterization of the dynamics.

extended the chaotic dynamics. Thus, even the minimal amount of space changes in a non-trivial way the dynamical properties as shown by the small lattices used in Fig. 3. However, the result is not exclusive of such small domains.

A quantitative measure of the degree of chaotic motion is provided by the estimation of the Lyapunov exponent $\left(\lambda_{L}\right)$, i.e. the rate of divergence of nearby trajectories. In this way, a system is called chaotic if at least one Lyapunov exponent is positive. For the uncoupled map (1) we estimate it by means of the expression (see Schuster (1989) :

$\lambda_{L}=\lim _{n \rightarrow \infty} \frac{1}{n} \sum_{t=1}^{n} \ln \left|\frac{\partial F\left(N_{t}\right)}{\partial N}\right|$.

eqn 8

On the other hand, when we are dealing with the CML counterparts we estimate the Lyapunov exponent from the local time series by means of the Wolf et al. (1985) algorithm:

$\lambda_{L}=\frac{1}{r \tau}\left\{\sum_{t=1}^{r} \ln [\Theta(t, \tau)]\right\}$

eqn 9 $r$ being the number of sampled points and

$\Theta(t, \tau)=\frac{\left\|N(t+\tau)-N^{\prime}(t+\tau)\right\|}{\left\|N(t)-N^{\prime}(t)\right\|}$.

eqn 10

Figure 4 shows the Lyapunov exponent for different $\beta$-values for the Hassell map and the corresponding spatially extended model (6). Not only are there more positive exponents for the spatial counterparts, but also there are higher values, indicating a larger sensitivity to initial conditions in the dynamics. An indication should be given here when looking at Fig. 4. For the CMLs there are no negative values. This is because the Wolf algorithm ( 9 and 10) only discriminates between zero values (fixed points and limit cycle attractors) and positive values for the chaotic attractors.

Two important results emerge from our study. In the first place, the qualitative dynamics shown by a population is not only perfectly determined by taking into account all the parameter values describing its biological growth, regulation, dispersal and interactions. Even if all these parameters are well known 
260

Spatially induced bifurcations
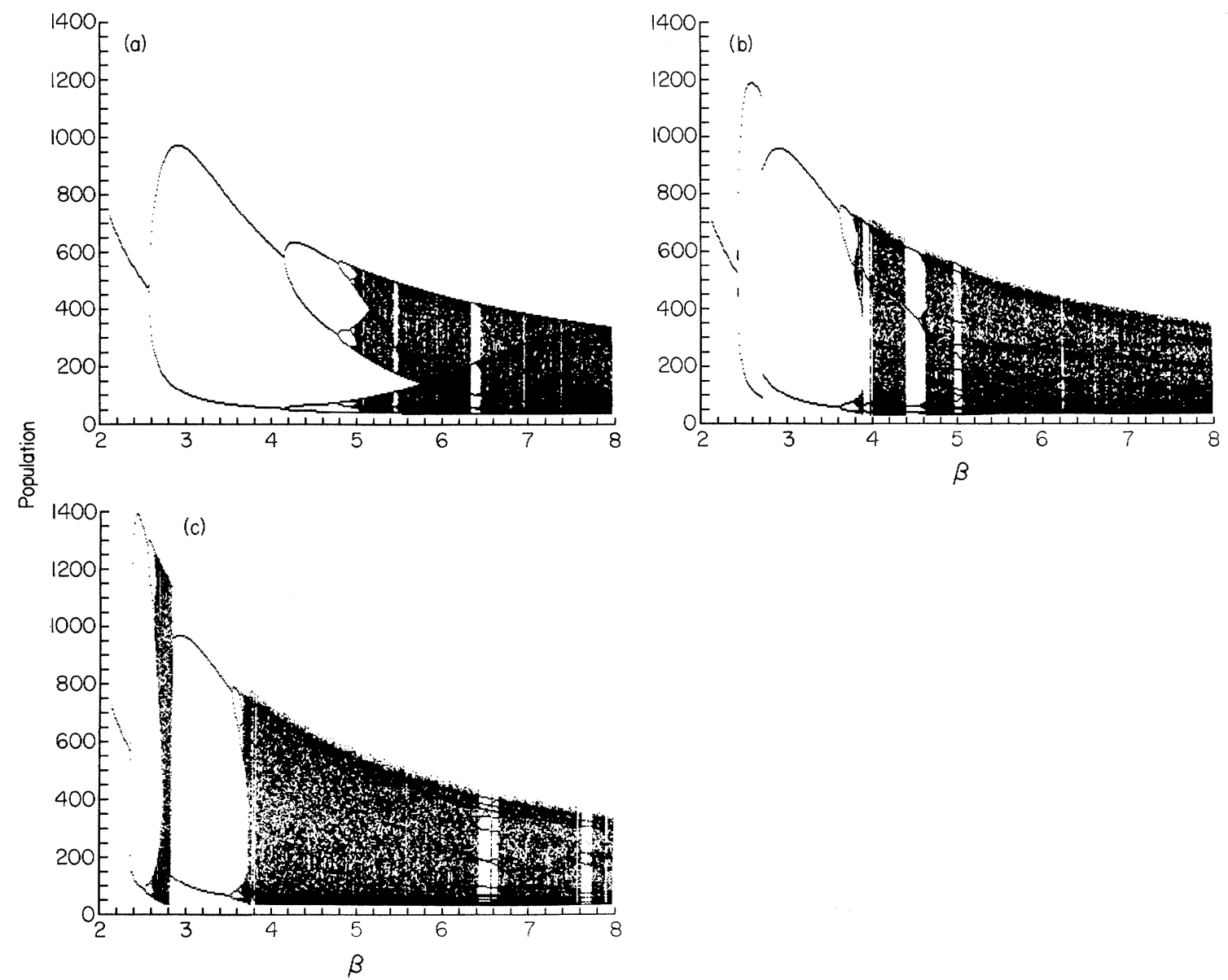

Fig. 3. Bifurcation diagrams. The local population density is plotted vs. parameter $\beta$ after 1000 transients are ruled out. (a) The Hassell map (equation 1) with $\lambda=60$ and $\alpha=0.01$; (b) the spatial counterpart with $D=0.02$ and lattice size $4 \times 4$; (c) the same, but for a lattice of $5 \times 5$. As shown, the larger the spatial domain, the more widely extended the chaotic motion: bifurcations take place for lower $\beta$-values.

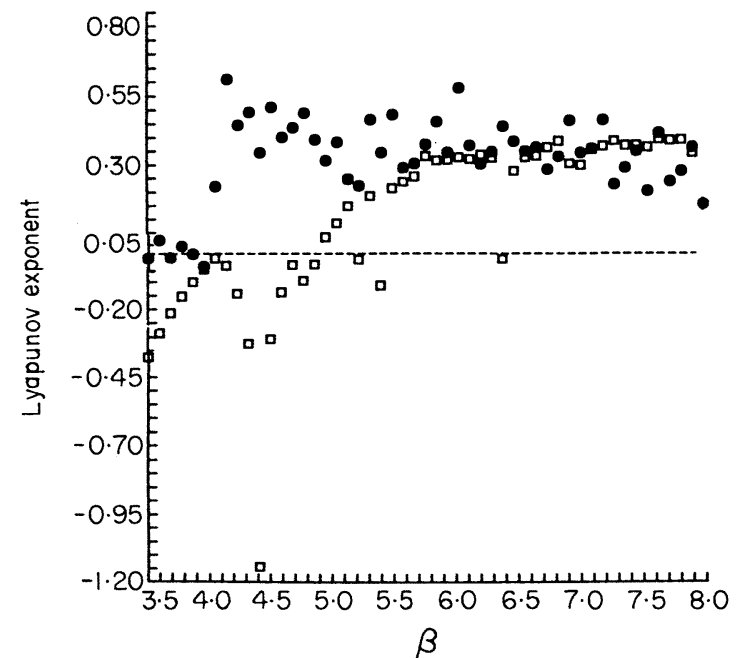

Fig. 4. Lyapunov exponents vs. $\beta$ for model $1(\square)$, and the same but for the local dynamics of the spatially extended counterpart (@). In the latter case, positive exponents indicating sensitive dependence on initial conditions are more common and in general they have a higher value. The over parameter values are as follows: for the map (equation (1)), $\lambda=60$ and $\alpha=0 \cdot 01$. For the CML the same as before with a lattice size $(L)$ of $7 \times 7$ and a diffusion rate $(D)=0.01$. we can see different dynamics depending on the size of the spatial domain in which this population evolves. In the second place and as a consequence, inferences about the underlying dynamics of a field (and so spatially extended) population are not possible by fitting a simple uncoupled model to the census data.

The same stability analysis carried out for the map (1) in the above section can be made for the CML (6). In this way we obtain the isocline defining the transition of the stable solution to the unstable domain (see Appendix). In Fig. 5 we can see in discontinuous lines such isoclines in three examples of the spatial model with diffusion rates of $0.02,0.08$ and $0 \cdot 2$, respectively. For comparison, the two isoclines of the Hassell map represented in Fig. 1 are also superimposed in continuous lines delimiting damped oscillations and stable limit cycles. As can be observed, the stability boundaries in the phase space are modified when space is taken into account. They are shifted toward the lower left side of the parameter space, indicating that bifurcations are reached for lower parameter values. If we superimpose the experimental points of Hassell, Lawton \& May's (1976) 
261

J. Bascompte \& R.V. Solé

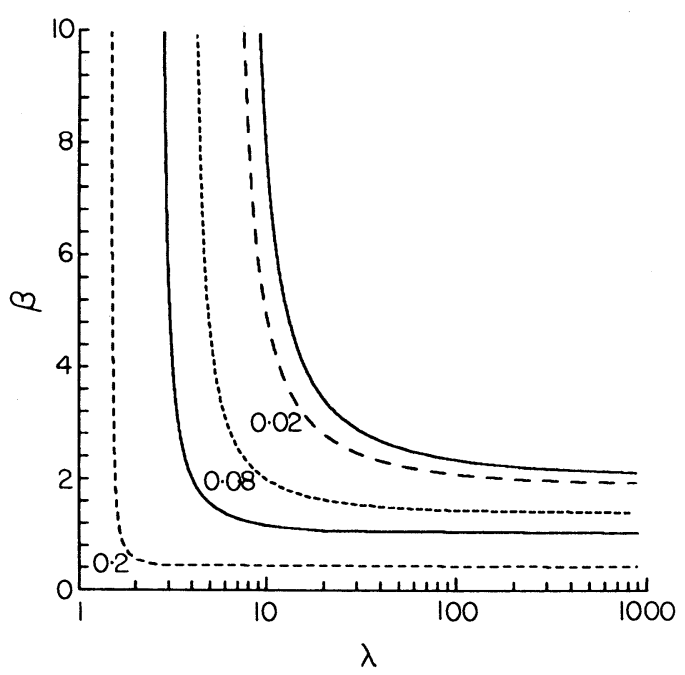

Fig. 5. Discontinuous isoclines: stability boundaries delimiting stable from unstable domains in the parameter space for the CML (equation 6) with diffusion rates of $0.02,0.08$ and $0 \cdot 2$, respectively from top to bottom (see Appendix). The two isoclines for the uncoupled map (equation 1) delimiting damped oscillations and stable limit cycles are superimposed in continuous lines (see Fig. 1).

work represented in Fig. 1, we would have to change our diagnostic of stability of those populations.

The effect of the lattice size on the coexistence and stabilization of populations is relative to their diffusion rates (Solé, Bascompte \& Valls 1992b). Due to this close relationship between parameters, similar results are provided by the study of the effects of the diffusion rate on the dynamics. It is well known from physical and chemical systems that the coupling of local dynamics by means of diffusion may cause instability (Kuramoto 1984). Such 'diffusion-induced chaos' seems to be a widely extended property of ecological systems when space is introduced. Chaos appears for a wide range of parameter combinations and, hence, it becomes structurally stable (Solé \& Valls 1992).

The effect of the diffusion rate on the qualitative behaviour of the Hassell CML (6) is shown in Fig. 6. A bifurcation diagram of model 6 has been represented for a lattice size of $5 \times 5$ when diffusion increases from 0 to $0 \cdot 055$. Note that when diffusion is 0 , the lattice points are uncoupled and there is no spatial effect. In this situation we can see that for the present parameter combination, there is steady state. As $D$ is progressively enlarged, there is a well-defined bifurcation scenario in which chaos beyond a characteristic $D$-value is reached. This is a good example of diffusion-induced chaos because, as noted above, this parameter set $(\lambda, \beta)$ corresponds to a stationary dynamic for the uncoupled model (1). This result is in agreement with the finding by Vance (1984) that dispersal can cause oscillations in previously stable systems.

\section{Discussion}

The main result achieved in this paper is that space has a key effect in controlling the dynamics of the populations that diffuse into it. As has been shown, the larger the lattice size, the more unstable the motion. However, since we have now been considering local dynamics, i.e. that corresponding to a lattice point, the question now is: Will we detect such an effect from field studies? As the bulk of the data record involves population census over a given area, they would be closer to global dynamics than local ones. Thus, we will now consider the effect of enlarging the space with regard to the global motion, i.e. the motion in which we consider every time-step the sum of individuals in all the spatial points. Figure 7 shows such

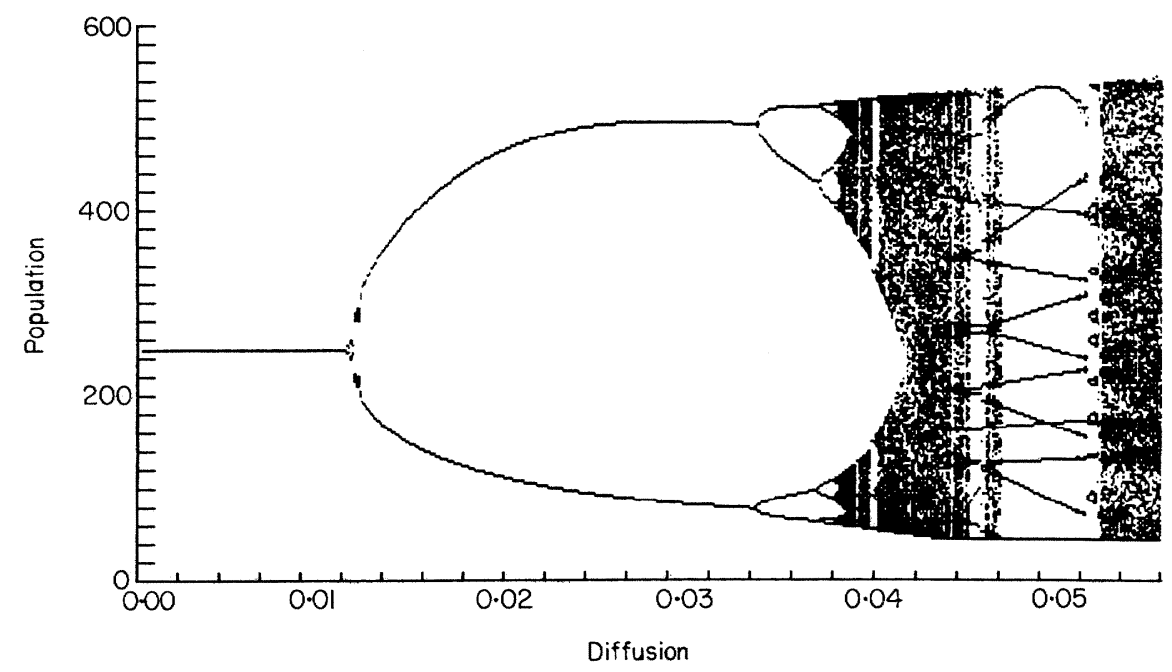

Fig. 6. The diffusion-induced bifurcations for the Hassell CML (6) is shown with $\lambda=30, \alpha=0 \cdot 01, \beta=2.7$ and $L=5 \times 5$. Local population density is plotted vs. diffusion rate $(D)$. 
262

Spatially induced bifurcations

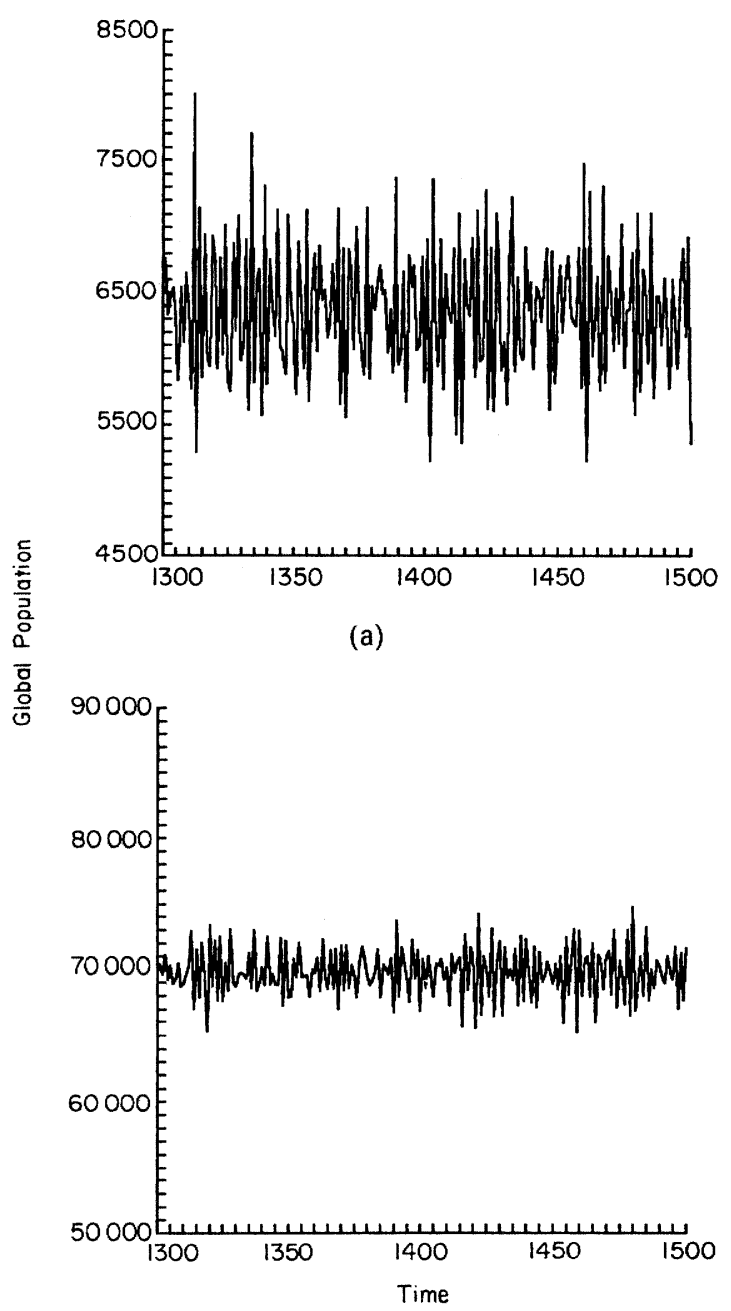

(b)

Fig. 7. Global dynamics for the CML (equation 6) with $\alpha=0 \cdot 01, \lambda=30, \beta=10, D=0 \cdot 02$, and $L$ is $15 \times 15$ (a) and $45 \times 45$ (b).

temporal data for lattice sizes of $15 \times 15$ (7a) and $45 \times 45$ (7b). As shown, as the spatial domain grows, global populations are more similar to that expected for a steady state with added noise. This fact, known as chaotic stability (Solé, Bascompte \& Valls 1992a) makes the characterization of chaos from field studies even more difficult (Solé \& Valls 1992). This implies that the spatial scale is important in order to achieve information on the way in which the ecosystems are organized. Patterns observed at a particular spatial scale cannot be the same at another hierarchical level. Note that the effect of lattice size on the dynamics can be the opposite, depending on the scale of observation: when we are considering local dynamics, the larger the spatial domain, the more unstable the motion. When, on the other hand, we are dealing with global dynamics, the larger the spatial domain, the more steady-state-like the changes in populations.

The idea reported here that the kind of dynamics shown by a population depends on environmental factors and is not single is well known from field studies. Different populations of the same species often have distinct dynamics. In particular, a great number of species have been reported cycling at the northern part of their range, while their dynamics are stationary at the southern part, e.g. snowshoe hare in America (Wolff 1980), and voles and other microtine rodents in Europe (Hanski 1987; Hanski, Hansson \& Henttonen 1991; Erlinge 1987). Several suggestions have been made to explain this difference in dynamical behaviour between geographical populations.

1. The structure of the food web. In the north, where populations show a cyclic variation in density, the food web is a simple chain, while in the south there is a greater diversity of both prey and predators and, therefore, more complex interactions (Hanski 1987). Furthermore, or as a consequence, there is a different stability effect between specialist predators (to the north, driving the cycle) and generalist ones (to the south, having a stabilizing effect) (Hanski et al. 1991; Anderson \& Erlinge 1977).

2. The size and continuity of the habitat. There is a difference between the larger forested areas in the north and the fragmented habitats due to human activity in the southern part of the range, differences that may be related to the latitudinal dynamical gradient (Wolff 1980).

Of course, these explanations are not incompatible and there is a general consensus between the authors cited above to the effect that there is no single operating mechanism. Here we are interested in the second explanation proposed, that of habitat size. As additional empirical support, there are a large number of observations of mice populations that fluctuate in their natural habitats, but not in small islands or artificially enclosed areas, confirming the importance of dispersal in the maintenance of multiannual cycles in density (Vance 1984; Gaines, Vivas \& Baker 1979; Tamarin 1978; Krebs et al. 1973). For a discussion of the behavioural mechanism of dispersal see Bekoff (1977). The theoretical results presented in this paper are in total agreement and provide an explanatory physical mechanism for such observations. In this way, the evidence of spatially induced bifurations gives us a theoretical framework from which we can characterize some field trends in population changes, helping us to focus attention on the role of space in the dynamics, stability and persistence of populations.

\section{Acknowledgements}

This study was supported by a grant from UPC No. PR9119.

\section{References}

Andersson, M. \& Erlange, S. (1977) Influence of predation in rodent populations. Oikos, 29, 591-597. 
J. Bascompte \& R.V. Solé
Bekoff, M. (1977) Mammalian dispersal and the ontogeny of individual behavioral phenotypes. American Naturalist, 111, 715-732.

Berryman, A.A. \& Millstein, J.A. (1989a) Are ecological systems chaotic: and if not why not? Trends in Ecology and Evolution, 4, 26-28.

Berryman, A.A. \& Millstein, J.A. (1989b). Letters to the editor. Trends in Ecology and Evolution, 4, 240.

Boerlijst, M.C. \& Hogeweg, P. (1991) Spiral wave structure in pre-biotic evolution: hypercycles stable against parasites. Physica D, 48, 17-28.

Comins, H.N., Hassell, M.P. \& May, R.M. (1992) The spatial dynamics of host-parasitoid systems. Journal of Animal Ecology, 61, 735-748.

Czárán, T. \& Bartha, S. (1992). Spatiotemporal dynamic models of plant populations and communities. Trends in Ecology and Evolution, 7, 38-42.

den Boer, P.J. (1987) Detecting density dependence. Trends in Ecology and Evolution, 2, 77-78.

Erlinge, S. (1987) Predation and noncyclity in a microtine population in southern Sweden. Oikos, 50, 347-352.

$\rightarrow$ Gaines, M.S., Vivas, A.M. \& Baker, C.L. (1979) An experimental analysis of dispersal in fluctuating vole populations: demographic parameters. Ecology, 60, 814-828.

Hanski, I. (1987) Populations of small mammals cycle-unless they don't. Trends in Ecology and Evolution, 2, 55-56.

Hanski, I., Hansson, L. \& Henttonen, H. (1991) Specialist predators, generalist predators and the microtine rodent cycle. Journal of Animal Ecology, 60, 353-367.

Hassell, M.P. (1975) Density-dependence in single-species populations. Journal of Animal Ecology, 44, 283-295.

Hassell, M.P., Lawton, J.N. \& May, R.M. (1976) Patterns of dynamical behaviour in single-species populations. Journal of Animal Ecology, 45, 471-486.

Hassell, M.P., Comins, H.N. \& May, R.M. (1991) Spatial structure and chaos in insect population dynamics. Nature, 353, 255-258.

Kaneko, K. (1989) Spatiotemporal chaos in one- and twodimensional coupled map lattices, Physica D, 37, 60-82.

Kaneko, K. (1990) Supertransients, spatiotemporal intermittency and stability of fully developed spatiotemporal chaos. Physics Letters A, 149, 105-112.

Kaneko, K. (1992) Overview of coupled map lattices. Chaos, 2, 279-282.

Kapral, R. (1985) Pattern formation in two-dimensional arrays of coupled, discrete-time oscillators. Physical Review A, 31, 3868-3879.

Kapral, R., Oppo, G. \& Brown, D.B. (1987) Phase separation and growth in a two-variable discrete model. Physica A, 147, 77-89.

Krebs, C.J., Gaines, M.S., Keller, B.L., Myres, J.H. \& Tamarin, R.H. (1973) Population cycles in small rodents. Science, 179, 35-41.

Kuramoto, Y. (1984) Chemical Oscillations, Waves and Turbulence. Springer, Berlin.

Lomnicki A. (1989) Avoiding chaos. Letters to the editor. Trends in Ecology and Evolution, 4, 239.

Mani, G.S. (1989) Avoiding chaos. Letters to the editor. Trends in Ecology and Evolution, 4, 239-240.

$\rightarrow$ May, R.M. (1974) Biological populations with nonoverlapping generations: stable points, stable cycles and chaos. Science, 186, 645-647.

May, R.M. (1976) Simple mathematical models with very complicated dynamics. Nature, 261, 459-467.

$\rightarrow$ May, R.M. \& Oster, G.F. (1976) Bifurcations and dynamic complexity in simple ecological models. American Naturalist, 110, 573-599.

Moloney, K.A., Levin, S.A., Chiariello, N.R. \& Buttel, L. (1992) Pattern and scale in a serpentine grassland. Theoretical Population Biology, 41, 257-276.

Morris, W.F. (1990) Problems in detecting chaotic behavior in natural populations by fitting simple discrete models. Ecology, 71, 1849-1862.

$\rightarrow$ Mueller, L.D. \& Ayala, F.J. (1981) Dynamics of singlespecies population growth: stability or chaos? Ecology, 62, 1148-1154.

Nisbet, R., Blythe, S., Gurney, B., Metz, H. \& Stockes, K. (1984) Avoiding chaos. Letters to the editor. Trends in Ecology and Evolution, 4, 238-239.

Pimm, S.L. (1991) The Balance of Nature? Chicago University Press.

Sabelis, M.W., Diekman, O. \& Jansen, U.A.A. (1991) Metapopulation persistence despite local extinction : predatorprey models of the Lotka-Volterra type. Biological Journal of the Linnean Society, 42, 267-283.

Schaffer, W.M. \& Kot, M. (1986) Chaos in ecological systems: the coals that Newcastle forgot. Trends in Ecology and Evolution, 1, 58-63.

Schuster, H.G. (1989) Deterministic Chaos. VCH Publishers, Germany.

Solé, R.V. (1991) Strange attractors, spatiotemporal chaos and criticality in complex biosystems. $\mathrm{PhD}$ thesis, Universitat Politècnica de Catalunya, Spain.

Solé, R.V. \& Valls, J. (1992) On structural stability and chaos in biological systems. Journal of Theoretical Biology, 155, 87-102.

Solé, R.V., Bascompte, J. \& Valls, J. (1992a) Nonequilibrium dynamics in lattice ecosystems: chaotic stability and dissipative structures. Chaos, 2, 387-395.

Solé, R.V., Bascompte, J. \& Valls, J. (1992b) Stability and complexity of spatially extended two-species competition. Journal of Theoretical Biology, 159, 469-480.

Solé, R.V., Valls, J. \& Bascompte, J. (1992) Spiral waves, chaos and multiple attractors in lattice models of interacting populations. Physics Letters A, 166, 123-128.

Sugihara, G. \& May, R.M. (1991) Nonlinear forecasting as a way of distinguishing chaos from measurement error in time series. Nature, 344, 734-741.

Tamarin, R.H. (1978) Dispersal, population regulation and $\mathrm{K}$-selection in field mice. American Naturalist, 112, 545555.

Taylor, A.D. (1990) Metapopulations, dispersal, and predator-prey dynamics: an overview. Ecology, 71, 429-433.

$\rightarrow$ Thomas, W.R., Pomeranz, M.J. \& Gilpin, M.E. (1980) Chaos, asymmetric growth and group selection for dynamical stability. Ecology, 61, 1312-1320.

Turing, A. (1952) The chemical basis of morphogenesis. Philosophical Transactions of the Royal Society of London, 237, 37-72.

Vance, R.V. (1984) The effect of dispersal on population stability in one-species, discrete space population growth models. American Naturalist, 123, 230-254.

Wolf, A., Swift, J.B., Swinney, H.L. \& Vastano, J.A. (1985) Determining Lyapunov exponents from a time series. Physica $D, 16,285-317$.

$\rightarrow$ Wolff, J.O. (1980) The role of habitat patchiness in the population dynamics of snowshoe hares. Ecological Monographs, 50, 111-130.

Received 11 December 1992; revision received 25 March 1993

\section{Appendix}

The stability analysis for the Hassell map (model 1) carried out in the text allows us to show the stability domains in the phase space (see also Hassell 1975). Here we develop the same stability analysis but for the corresponding coupled map lattice. This permits us to represent the isoclines for the spatially extended 
264

Spatially induced bifurcations system in Fig. 5. The stability domains are different for the CML.

The solution of the steady state for the spatially extended counterpart is stable provided that (Kapral 1985; Kapral, Oppo \& Brown 1987)

$$
\left|\frac{\partial F\left(N^{*}\right)}{\partial N}+D \Theta(\mathbf{k})\right|<1
$$

$D$ being the diffusion rate, $\mathbf{k}=k, l$ is proportional to the inverse of the wavelength over the lattice and $\Theta(\mathbf{k})$ is the so called wavevector, a given function of $\mathbf{k}=k$, $l$. In this way, $\mathbf{k}$ is related to the wavelength of the perturbation from the spatially homogeneous situation. The problem is similar to that for the characterization of the spatial symmetry breaking in the Turing structures (Turing 1952). In particular, the function takes the expression :

$\Theta(\mathbf{k})=\Theta(k, l)=4\left\{\cos \frac{\pi(k+l)}{L} \cos \frac{\pi(k-l)}{L}-1\right\}$ eqn A2

where $L$ is the lattice size.

It is easily seen that for the trivial case $\mathbf{k}=(0,0)$ the wavelength would be $L / 0$, i.e. infinite, which means that there is no departure from the homogeneous state and, as a consequence, space has no importance. In this case, $\Theta(\mathbf{k})=0$ and there is only the first term in (equation A1), as for the uncoupled map.

In the spatially extended system, stability boundaries are obtained from the limiting case $\mathbf{k}_{c}$ where $k=l=L / 2$ corresponding to the smallest wavelength. Replacing this value in equation (A2) we obtain $\Theta\left(\mathbf{k}_{c}\right)=-8$. Taking into account this result and equation (A1), we find that the isocline will be given by the following equality:

$\frac{\partial F\left(N^{*}\right)}{\partial N}-8 D=-1$

eqn $\mathrm{A} 3$

that for the Hassell map runs

$1-\beta\left(1-\lambda^{-1 / \beta}\right)-8 D=-1$ eqn A4

rewriting equation (A4) in an explicit way we obtain

$\lambda=\exp \left[-\beta \ln \left(1+\frac{8 D-2}{\beta}\right)\right]$

eqn $\mathrm{A} 5$

The last expression provides the isocline dividing the stable from the unstable domain in the parameter space for a CML with a given diffusion rate. Again, it can be observed that for the critical case of $D=0$ we have a set of $L \times L$ uncoupled maps and equation (A5) coincides with equation (3). 